DOI:

10.1038/nrn2138

NEUROGENESIS

\title{
Single stress shortens survival
}

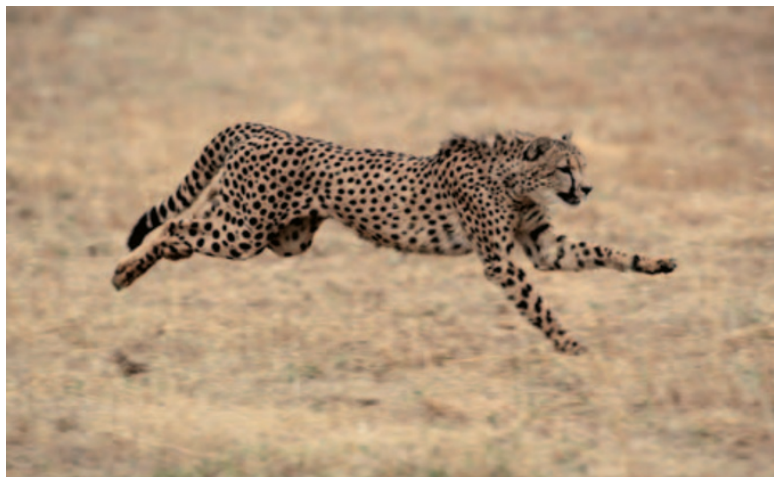

How much stress does it take to kill a new neuron? Thomas and colleagues show that, in rats, one acute exposure to psychosocial stress reduces both short- and long-term survival of newborn hippocampal neurons.

Stress is a predisposing factor for depression, and animal models have indicated that chronic stress in particular might have a role in the aetiology of affective disorders by inhibiting neurogenesis in the hippocampal dentate gyrus. However, whether acute stress also influences neurogenesis is unclear. Thomas et al. exposed adult rats to the naturalistic experience of social defeat by allowing an intruder rat to enter the territory of a colony of rats including an aggressive dominant male, and determined the effects of a single, acute episode of this psychosocial stressor on the different stages of neurogenesis.

To label newborn cells, the researchers injected rats with the thymidine analogues CldU and IdU, or with BrdU, before the stress exposure. The timing of the stressor varied from immediately post-injection to 1 week after the last injection; this experimental setup allowed them to distinguish the effects of acute stress on the proliferation of hippocampal cells and on their immediate, shortterm and long-term survival.

CldU, injected one day before stress exposure so it would label cells that were proliferating at the time of the stressor, was incorporated to the same extent in the dentate gyrus of stressed and control rats. The labelling of IdU, injected 2-3 days before the stressor, was also similar in dentate gyrus cells from stressed and control rats. These findings indicate that the psychosocial stressor had not affected hippocampal cell proliferation or their immediate survival. By contrast, when the incorporation of $\mathrm{BrdU}$ - given in the 3 days prior to stress exposure - was measured 1 week after the stressor, the researchers found that it was $66 \%$ lower in stressed rats than in control rats, indicating that psychosocial stress had reduced short-term survival of newborn cells by a third.

In order to investigate long-term neuronal survival, the researchers exposed a third group of rats to the stressor 1 week after the last injection with BrdU, allowing the newborn cells to differentiate. Three weeks after stress, 33\% fewer cells were positive for both BrdU and the neuronal cell marker NeuN in the dentate gyrus of stress-exposed compared with control rats, demonstrating decreased long-term survival of neurons.

In this elegantly designed study, Thomas et al. revealed a temporal aspect to the effect of stress on neuronal survival. The authors speculate that a single exposure to stress may leave proliferation unaffected but render the hippocampal environment less supportive for the subsequent survival of the newborn neurons. If this is indeed the case, future research may probe the possibility of manipulating the neuronal environment in order to promote the survival of new neurons after stress.

Leonie Welberg

ORIGINAL RESEARCH PAPER Thomas, R. M. et al. Acute psychosocial stress reduces cell survival in adult hippocampal neurogenesis without altering proliferation. J. Neurosci. 27, 2734-2743 (2007) 